\title{
Evaluation of a bacteriophage-typing scheme for Enterobacter cloacae
}

\author{
M. A. GASTON
}

Division of Hospital Infection, Central Public Health Laboratory, 61 Colindale Avenue, London NW9 5HT

\begin{abstract}
Summary. A set of 25 Enterobacter cloacae typing phages was evaluated. Of 384 test strains, $93.8 \%$ were lysed by at least one phage; the mean number of reactions/strain was 7.3. Discrimination between strains was satisfactory within the most frequent $O$ serotypes; 0.9 patterns/strain were found for strains of serotypes $\mathrm{O} 3$ and O8. Overall, 325 patterns were found. Phage patterns were completely reproducible when duplicates of strains were typed on the same day, but only $40 \%$ reproducible when repeated after 18 months. The combination of $O$ serology and phage typing discriminated well between hospital isolates.
\end{abstract}

\section{Introduction}

Enterobacter cloacae has been implicated in several outbreaks of nosocomial infection (Mayhall et al., 1979; Powell et al., 1980; John et al., 1982) but the extents of these episodes have been difficult to document because of lack of typing schemes. The discrimination provided by the $\mathrm{O}$-serotyping scheme described recently (Gaston et al., 1983) was fair, but further methods were required to subdivide the common $\mathrm{O}$ serotypes. The isolation and selection of a set of typing phages for $E$. cloacae has been described in a companion paper (Gaston, 1987). In this study, use of this set as a secondary typing method is evaluated.

\section{Materials and methods}

\section{Phages}

The 25 typing phages were selected from a candidate set of 76 phages isolated mainly from sewage (Gaston, 1987).

\section{E. cloacae strains}

A test series of 384 strains of $E$. cloacae was selected from a collection of 1600 clinical isolates. O serotyping was performed as described by Gaston et al. (1983).

\section{Routine phage typing}

Broth cultures of the test strains were diluted 1 in 200 and seeded on to 9-cm diameter phage-typing agar (PTA) plates (Gaston, 1987). Excess broth was removed with a pasteur pipette and the plates were dried for $30 \mathrm{~min}$ at room temperature. The phages were then applied at

Received 2 Jul. 1986; accepted 8 Dec. 1986. routine test dilution (RTD) with a multiloop applicator (Lidwell, 1959). Plates were incubated for $18 \mathrm{~h}$ at $32^{\circ} \mathrm{C}$ and the phage reactions were recorded. The strength of lysis was scored as: strong reactions, $>50$ plaques $(++)$; intermediate, $20-50$ plaques $(+)$; weak, 1-19 plaques $( \pm)$; or no reaction $(-)$.

\section{Reader variation}

Three readers who were experienced in recording phage reactions scored the reactions independently with 143 strains. Variations in their scoring were noted for each phage.

\section{Reproducibility on repeated typing}

(i) Thirty strains were typed twice on the same day to determine the immediate reproducibility of the typing set; (ii) 84 strains were retyped after storage for 18 months at room temperature. All strains had been subcultured at least once during that period.

\section{Associations of phage types with serotypes}

Associations were determined by comparing the reactivity of each phage with individual serotypes against its reactivity with all other serotypes. Fisher's Exact Probability test was used to determine whether the phages were positively or negatively associated with the most common serotypes (Croxton and Cowden, 1962).

\section{Results}

\section{Typing ability}

For the purposes of this analysis, only those reactions with $\geqslant 20$ plaques were considered to be positive. Of the 384 strains, $360(93.8 \%)$ were lysed 
by at least one of the 25 phages, and the mean number of reacting phages for each strain was 7.3. The percentages of strains of different serotypes which could be phage typed ranged from $90 \%$ for serotype $\mathrm{O} 27$ to $100 \%$ for several other serotypes (table I). The mean number of reactions of the strains of different serotypes varied widely, from a mean of 2.6 phage reactions for strains of serotype $\mathrm{O} 27$ to a mean of 10.4 reactions for strains of serotype 019. Strains that were not serologically typable (O-NT or autoagglutinable) were usually sensitive to the typing phages, $84.8 \%$ of O-NT strains and $94.3 \%$ of autoagglutinable (AA) strains being typable.

Reactions of individual phages. These are displayed in table II. The proportion of strains giving strong lytic reactions ranged from $47 \cdot 1 \%$ for phage 7 to $6 \cdot 3$ for phage 21 . Twelve phages gave strong $(++)$ or intermediate $(+)$ reactions with one-third or more of the test strains and only two phages reacted with $<10 \%$ of strains.

Associations. The reactions of each phage with the ten most frequent serotypes were analysed. Nine serotypes showed at least one significant association. The number of associations varied but was particularly marked in strains of serotypes $\mathrm{O} 3$ and 08 . There were six positive associations in the 25 phages with strains of serotype $\mathrm{O} 3$ and five negative associations. Phage 25 was unique in that it was the only serotype specific phage, lysing 32 of the 53 serotype $\mathrm{O} 3$ strains. Eight phages were positively associated with serotype 08 strains. Autoagglutinable strains showed an increased sensitivity to phages $15,18,19,21$ and 23 .

Table I. Distribution of the phage reactions by $O$ serotype

\begin{tabular}{|c|c|c|c|}
\hline O serotype & $\begin{array}{l}\text { Number of } \\
\text { strains } \\
\text { examined }\end{array}$ & $\begin{array}{c}\text { Mean number of } \\
\text { phage reactions } \\
\text { per strain }\end{array}$ & $\begin{array}{l}\text { Percentage of } \\
\text { strains typable }\end{array}$ \\
\hline 1 & 21 & $6 \cdot 2$ & $90 \cdot 5$ \\
\hline 3 & 53 & $8 \cdot 1$ & $94 \cdot 3$ \\
\hline 4 & 10 & $5 \cdot 7$ & $100 \cdot 0$ \\
\hline 8 & 45 & 8.9 & 95.6 \\
\hline 9 & 16 & 7.8 & $100 \cdot 0$ \\
\hline 12 & 11 & $5 \cdot 2$ & $100 \cdot 0$ \\
\hline 13 & 23 & $7 \cdot 0$ & 95.7 \\
\hline 16 & 17 & 6.9 & $100 \cdot 0$ \\
\hline 19 & 10 & 10.4 & $100 \cdot 0$ \\
\hline 27 & 10 & $2 \cdot 6$ & $90 \cdot 0$ \\
\hline \multirow{4}{*}{$\begin{array}{c}\text { AA } \\
\text { O-NT } \\
\text { All } \\
\text { strains }\end{array}$} & 35 & $8 \cdot 7$ & $94 \cdot 3$ \\
\hline & 46 & 5.8 & 84.8 \\
\hline & & & \\
\hline & 384 & $7 \cdot 3$ & 93.8 \\
\hline
\end{tabular}

Table II. Lytic reactions of the 25 typing phages on 384 distinct strains of $E$. cloacae

\begin{tabular}{|c|c|c|c|c|}
\hline \multirow{2}{*}{$\begin{array}{c}\text { Phage } \\
\text { no. }\end{array}$} & \multicolumn{4}{|c|}{ Number of strains $(\%)$ with the indicated reaction } \\
\hline & - & \pm & + & ++ \\
\hline 1 & $202(52 \cdot 6)$ & $20(5 \cdot 2)$ & $29(7 \cdot 6)$ & $133(34 \cdot 6)$ \\
\hline 2 & $185(48 \cdot 2)$ & $10(2 \cdot 6)$ & $10(2 \cdot 6)$ & $179(46 \cdot 6)$ \\
\hline 3 & $226(58.9)$ & $21(5 \cdot 5)$ & $19(4.9)$ & $118(30 \cdot 7)$ \\
\hline 4 & $184(47 \cdot 9)$ & $7(1 \cdot 8)$ & $17(4 \cdot 4)$ & $176(45 \cdot 8)$ \\
\hline 5 & $243(63 \cdot 3)$ & $5(1 \cdot 3)$ & $12(3 \cdot 1)$ & $124(32 \cdot 3)$ \\
\hline 6 & $239(62 \cdot 2)$ & $16(4 \cdot 2)$ & $26(6 \cdot 8)$ & $103(26 \cdot 8)$ \\
\hline 7 & $173(45 \cdot 1)$ & $6(1 \cdot 6)$ & $24(6 \cdot 3)$ & $181(47 \cdot 1)$ \\
\hline 8 & $203(52.9)$ & $6(1 \cdot 6)$ & $16(4 \cdot 2)$ & $159(41 \cdot 4)$ \\
\hline 9 & $297(77 \cdot 3)$ & 0 & $4(1 \cdot 0)$ & $83(21 \cdot 6)$ \\
\hline 10 & $232(60 \cdot 4)$ & $5(1 \cdot 3)$ & $15(3.9)$ & $132(34 \cdot 4)$ \\
\hline 11 & $230(59.9)$ & $8(2 \cdot 1)$ & $16(4 \cdot 2)$ & $130(33.9)$ \\
\hline 12 & $215(56 \cdot 0)$ & $14(3 \cdot 6)$ & $26(6.8)$ & $129(33.6)$ \\
\hline 13 & $274(71 \cdot 4)$ & $14(3 \cdot 6)$ & $17(4 \cdot 4)$ & $79(20 \cdot 6)$ \\
\hline 14 & $291(75 \cdot 8)$ & $10(2 \cdot 6)$ & $9(2 \cdot 3)$ & $74(19 \cdot 3)$ \\
\hline 15 & $303(78.9)$ & $6(1 \cdot 6)$ & $9(2 \cdot 3)$ & $66(17 \cdot 2)$ \\
\hline 16 & $279(72 \cdot 7)$ & $28(7 \cdot 3)$ & $21(5 \cdot 5)$ & $56(14 \cdot 6)$ \\
\hline 17 & $295(76 \cdot 8)$ & $8(2 \cdot 1)$ & $5(1 \cdot 3)$ & $76(19.8)$ \\
\hline 18 & $304(79 \cdot 2)$ & $3(0.8)$ & $6(1 \cdot 6)$ & $71(18 \cdot 5)$ \\
\hline 19 & $236(61 \cdot 5)$ & $5(1 \cdot 3)$ & $15(3.9)$ & $128(33 \cdot 3)$ \\
\hline 20 & $280(72.9)$ & $4(1 \cdot 0)$ & $8(2 \cdot 1)$ & $92(24.0)$ \\
\hline 21 & $342(89 \cdot 1)$ & $14(3 \cdot 6)$ & $4(1 \cdot 0)$ & $24(6 \cdot 3)$ \\
\hline 22 & $324(84 \cdot 4)$ & $10(2 \cdot 6)$ & $19(4.9)$ & $31 \quad(8 \cdot 1)$ \\
\hline 23 & $335(87 \cdot 2)$ & $8(2 \cdot 0)$ & $7(1 \cdot 8)$ & $34(8.9)$ \\
\hline 24 & $329(85 \cdot 7)$ & $3(0 \cdot 8)$ & $4(1.0)$ & $48(12 \cdot 5)$ \\
\hline 25 & $347(90 \cdot 4)$ & $5(1 \cdot 3)$ & $2(0.5)$ & $30 \quad(7.8)$ \\
\hline
\end{tabular}

,- No reaction;, $\pm 1-19$ plaques;,$+ 20-50$ plaques; ,$++>50$ plaques.

\section{Discrimination}

Distinct phage patterns were established by scoring only those reactions with $\geqslant 20$ plaques as positive. If the all-negative pattern (NT) is excluded, 325 distinct patterns were recognised in the 384 strains $(0.85$ patterns/strain). Only three patterns occurred on three or more occasions: phage 9 alone (five times), phages 19 and 20 (three times) and phage 15 alone (three times). Table III shows the distribution of distinct patterns for each of the frequent serotypes and for O-NT and AA strains. This table also shows the effect of applying different reaction-difference rules to the patterns obtained with the typing set. If strains were considered to be adequately separated by any single strong reaction difference there were 0.85 patterns/strain. However, if one strong reaction difference was ignored and at least two were required before strains were classified as distinct, the number of patterns distinguished by the typing set fell to $273(0.71$ patterns/strain) and if three reaction differences were required, the number of distinct patterns was $209(0 \cdot 54 /$ strain $)$. 
Table III. The effect of applying a reaction difference rule on the number of distinct patterns defined in 384 strains

\begin{tabular}{|c|c|c|c|c|c|}
\hline \multirow[b]{2}{*}{ O serotype } & \multirow{2}{*}{$\begin{array}{l}\text { Number of } \\
\text { strains }\end{array}$} & \multicolumn{4}{|c|}{$\begin{array}{c}\text { Number of distinct patterns for } \\
\text { stated number of reaction } \\
\text { differences }\end{array}$} \\
\hline & & 0 & 1 & 2 & 3 \\
\hline 1 & 21 & 18 & 18 & 15 & 14 \\
\hline 3 & 53 & 49 & 47 & 44 & 35 \\
\hline 4 & 10 & 7 & 7 & 6 & 5 \\
\hline 8 & 45 & 42 & 38 & 28 & 21 \\
\hline 9 & 16 & 15 & 15 & 12 & 9 \\
\hline 12 & 11 & 11 & 10 & 9 & 8 \\
\hline 13 & 23 & 19 & 18 & 15 & 12 \\
\hline 16 & 17 & 17 & 14 & 10 & 7 \\
\hline 19 & 10 & 10 & 9 & 9 & 9 \\
\hline 27 & 10 & 9 & 8 & 5 & 4 \\
\hline AA & 36 & 34 & 33 & 32 & 27 \\
\hline O-NT & 46 & 39 & 38 & 27 & 21 \\
\hline All strains & 384 & 325 & 273 & 209 & 142 \\
\hline
\end{tabular}

The discrimination achieved was satisfactory within the most frequent serotypes. The number of patterns/strain varied between $0 \cdot 7$ for serotype $\mathrm{O} 4$ strains to 1.0 for strains of serotype O19. In the four largest groups indistinguishable by serology, the mean number of patterns for $\mathrm{O} 3, \mathrm{O} 8$ and $\mathrm{AA}$ strains was 0.9 and for $\mathrm{O}-\mathrm{NT}$ strains was $0 \cdot 8$.

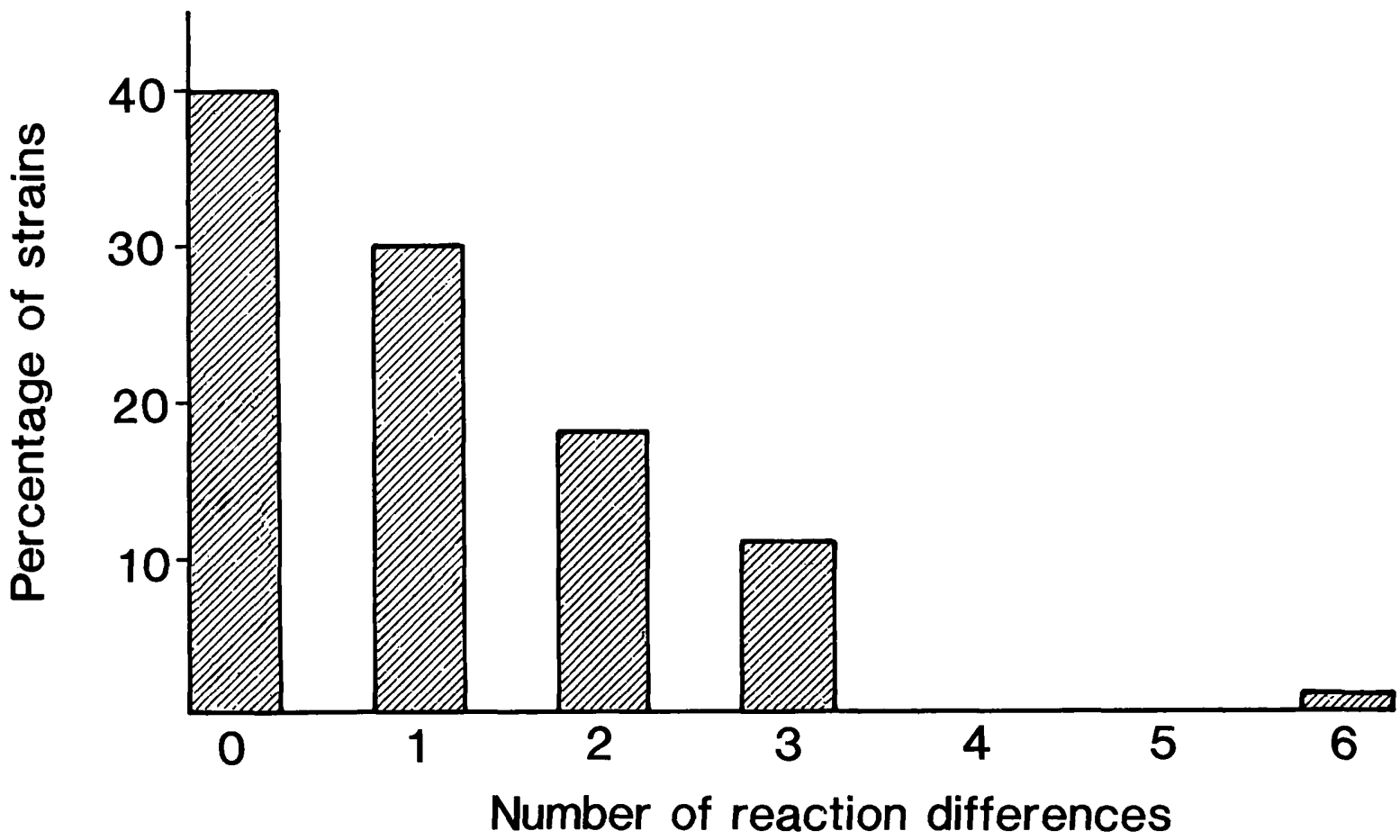

Figure. Reproducibility of phage typing after storage of strains; percentage of 84 strains giving the indicated number of reaction differences on retyping after storage for 18 months.

\section{Reproducibility}

Inter-observer variation. For the purposes of the evaluation, the readings of each of the three phage readers who took part in the analysis were given equal weight and the three readings of each plate were compared with each other. Hence there were $429(143 \times 3)$ plate comparisons and 10,725 comparisons of individual phage reactions.

Of the 429 plate comparisons, $375(87 \%)$ gave identical results, $28(7 \%)$ showed one strong reaction difference $(++$ to -$), 16(4 \%)$ revealed two reaction differences, and $10(2 \%)$ gave three or more reaction differences. A high proportion of the differences were due to incorrect identification of the phages by the reader rather than to errors in assessing the strength of reaction. The average number of strong reaction differences/phage was 3.7 and the range of differences expressed as percentages of the numbers of strong reactions varied between $0 \%$ for three phages and $12.9 \%$ for phage 9. This last phage produced small turbid plaques on PTA medium that were often difficult to read.

Duplicate plates from one broth. There were no strong reaction differences between the duplicated plates of 30 strains typed on the same day. The only differences recorded were between ++ and + reactions. 
Separate single colonies from one strain. Twenty distinct colonies from three strains were typed. No strong reaction differences were observed.

Variation of phage pattern on storage. Retyping 84 strains after storage for 18 months at room temperature on Dorset's egg medium showed that several strains had become autoagglutinable and their phage patterns differed markedly from the original, usually gaining two or three reactions. The reproducibility of typing the 84 strains tested is shown in the figure. The phage susceptibility patterns of only $40 \%$ of the strains were identical after this period but if two reaction differences were allowed before the cultures were classified as distinct, $88 \%$ of strains remained unchanged. Only phages 3 and 9 were $<90 \%$ reproducible.

Multiple isolates from the same patient. Thirtynine pairs of cultures from the same patient and with the same $O$ serotype were selected and typed on the same day. There were eight strong reaction differences $(++$ to -$), 0 \cdot 21 /$ strain, and seven moderate $(+$ to -$)$ reaction differences, $0 \cdot 18 /$ strain.

Variation in phage pattern during an outbreak. There were few outbreaks during the study period with which to evaluate the stability of the phage susceptibility patterns. In two of three well-defined episodes, which involved patient-to-patient spread of the index strains, the phage patterns of all isolates of each strain were identical. In a third, common source, outbreak, 46 of 48 isolates gave essentially identical phage patterns. The remaining two isolates were believed also to represent the index strain because of the epidemiological picture, and because they had similar antibiograms and flagellar antigens (unpublished results), but gave distinct phage patterns. One of these isolates was partially autoagglutinable in saline but retained sufficient type antigen to be detected (this was identical to that of the outbreak strain) and the other was completely autoagglutinable. These results suggest that the changes in phage pattern resulted from loss of $\mathrm{O}$ antigen.

\section{Discussion}

The bacteriophage typing scheme was developed to provide a secondary method of typing for $E$. cloacae. The phages were chosen primarily for their ability to subdivide the groups of strains which could not be divided by $\mathrm{O}$ serology, i.e., $\mathrm{O} 3(19.1 \%$ of nosocomial isolates), O8 (16.3\%), O-NT (12.3\%) and AA strains $(5.6 \%)$. The typing set discriminated well within these groups and provided a satisfactory method of subdividing most of the other $\mathrm{O}$ serotypes.

The poor reproducibility of typing patterns over a long time period may limit the application of the scheme to local situations. There are several factors that can adversely effect the reproducibility of phage typing. The initial ratio of active phage particles to bacterial cells is important (Bergan and Lystad, 1972). The susceptibility of cells also depends on their physiological state. Apparent variation in phage patterns can also occur due to inaccurate recording of the reactions. The poor reproducibility of the $E$. cloacae typing was probably due to a combination of these factors. The relative instability of $E$. cloacae phage patterns was comparable with the findings of other workers with phage-typing systems for Pseudomonas aeruginosa and staphylococci (Williams and Rippon, 1952; Sjoberg and Lindberg, 1968). Two phages, nos. 3 and 9, showed particularly poor reproducibility and their removal from the set would have greatly enhanced the reliability of the results.

The variation in the phage patterns of isolates that had been stored for a period of time may have been due, at least in part, to alterations in surface antigens of the strains. Several of the phages in the typing set appeared to be sensitive to changes in the amount of $O$ side chain present on the cell surface and strains that had become overtly autoagglutinable during storage showed altered susceptibility to the phages. The significant association of five phages with the autoagglutinable phenotype suggests that loss of $\mathrm{O}$ antigen may increase the availability of the attachment sites of these phages. The typing of clinical isolates and laboratory variants that failed to express $\mathrm{O}$-side chain polysáccharide (unpublished results) indicated that loss of $O$ antigen can significantly alter the phage-susceptibility pattern.

The strong associations between some phages and certain serotypes facilitated the identification of the $\mathbf{O}$ antigens of these strains from their phage types. These associations could limit the discrimination of the scheme in some instances. In pairs of isolates with similar phage patterns it may be difficult to determine whether the isolates were related or whether the similarities were due to the shared $\mathbf{O}$ antigens. In practice, confusions attributable to these associations were uncommon.

A number of phages with similar reactions included in the 25-phage set were selected by DTA3 because of their ability to discriminate between strains. This study has shown that much of the 
information provided by these phages was duplicated and therefore redundant. Accordingly seven of them were removed to form an 18-phage set, and this, rather than the 25-phage set, is currently recommended for typing clinical isolates.

\section{REFERENCES}

Bergan T, Lystad A 1972 Reproducibility in bacteriophage sensitivity pattern of Pseudomonas aeruginosa. Acta Pathologica et Microbiologica Scandinavica Section B 80: 345-350

Croxton F E, Cowden D J 1962 Applied general statistics, 2nd edn. Pitman and Sons, London, pp 686-689.

Gaston M A, Bucher C, Pitt T L 1983 O serotyping scheme for Enterobacter cloacae. Journal of Clinical Microbiology 18: 1079-1083.

Gaston M A 1987 Isolation and selection of a bacteriophage typing set for Enterobacter cloacae. Journal of Medical Microbiology 24: 285-290.

John J F, Sharbaugh R J, Bannister E R 1982 Enterobacter cloacae: bacteremia, epidemiology, and antibiotic resistance. Reviews of Infectious Diseases 4: 13-28.
The combination of $O$ serology and phage typing has proved to be a powerful tool for discrimination between hospital strains of $E$. cloacae and should contribute to the epidemiological analysis of nosocomial infection caused by this organism.

Lidwell O M 1959 Apparatus for phage-typing of Staphylococcus aureus. Monthly Bulletin of the Ministry of Health 18: 49-52.

Mayhall C G, Lamb V A, Gayle W E, Haynes B W 1979 Enterobacter cloacae septicemia in a burn center: epidemiology and control of an outbreak. Journal of Infectious Diseases 139: 166-171.

Powell J, Bureau M A, Pare C, Gaildry M L, Cabana D, Patriquin H 1980 Necrotizing enterocolitis. Epidemic following an outbreak of Enterobacter cloacae type 3305573 in a neonatal intensive care unit. American Journal Diseases of Children 134: 1152-1154.

Sjoberg L, Lindberg A A 1968 Phage typing of Pseudomonas aeruginosa. Acta Pathologica et Microbiologica Scandinavica 74: 61-68.

Williams R E O, Rippon J E 1952 Bacteriophage typing of Staphylococcus aureus. Journal of Hygiene 50: 320-353. 